Supplement of The Cryosphere Discuss., 9, 6495-6543, 2015

http://www.the-cryosphere-discuss.net/9/6495/2015/

doi:10.5194/tcd-9-6495-2015-supplement

(C) Author(s) 2015. CC Attribution 3.0 License.

(c) (i)

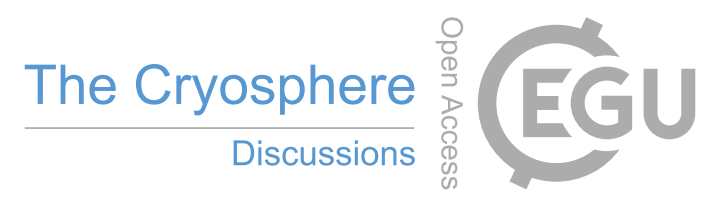

Supplement of

\title{
Characterizing Arctic sea ice topography using high-resolution IceBridge data
}

\author{
A. A. Petty et al. \\ Correspondence to: A. A. Petty (alek.a.petty@nasa.gov)
}

The copyright of individual parts of the supplement might differ from the CC-BY 3.0 licence. 
DMS Date: 20100405 DMS Time: $12253130 \quad 81.18 \mathrm{~N},-101.85 \mathrm{E}$
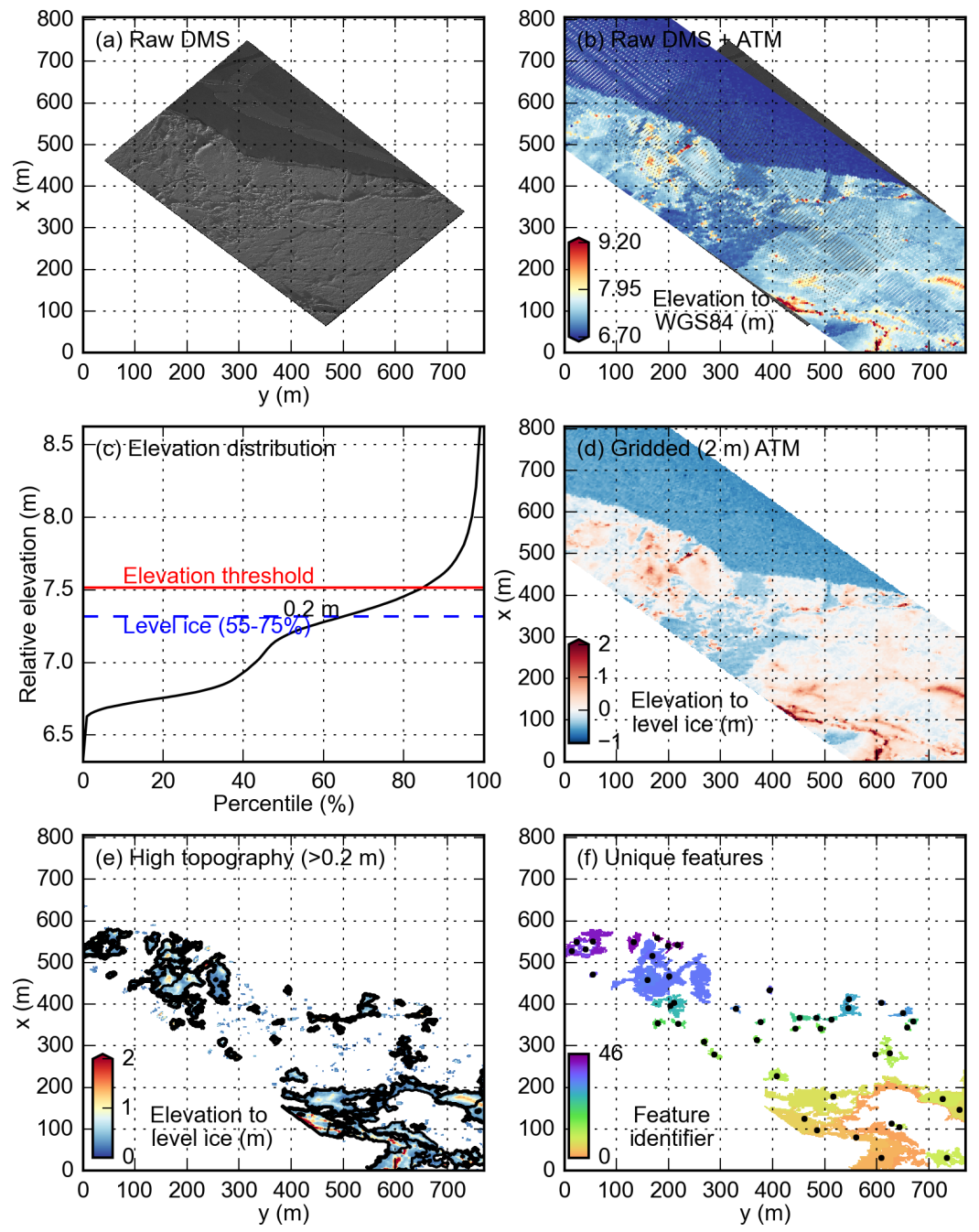
Figure S1. Example of the surface feature detection algorithm overlaid on a DMS image taken on the 5th April 2010 as highlighted by the green star in Figure 2. (a) DMS image; (b) Raw ATM data overlaid on the DMS image; (c) Elevation distribution for all ATM points within the section shown, where the blue line indicates the bounds of the calculated level ice surface and the red line indicates the feature height threshold; (d) Gridded (2 m) ATM elevation relative to the level ice surface; (e) Unique surface features $(>20 \mathrm{~cm})$ and their elevation relative to the level ice surface; (f) Unique surface feature identifier (features larger than $100 \mathrm{~m}^{2}$ ). 
DMS Date: 20110323 DMS Time: $17445601 \quad 73.02 \mathrm{~N},-146.78 \mathrm{E}$
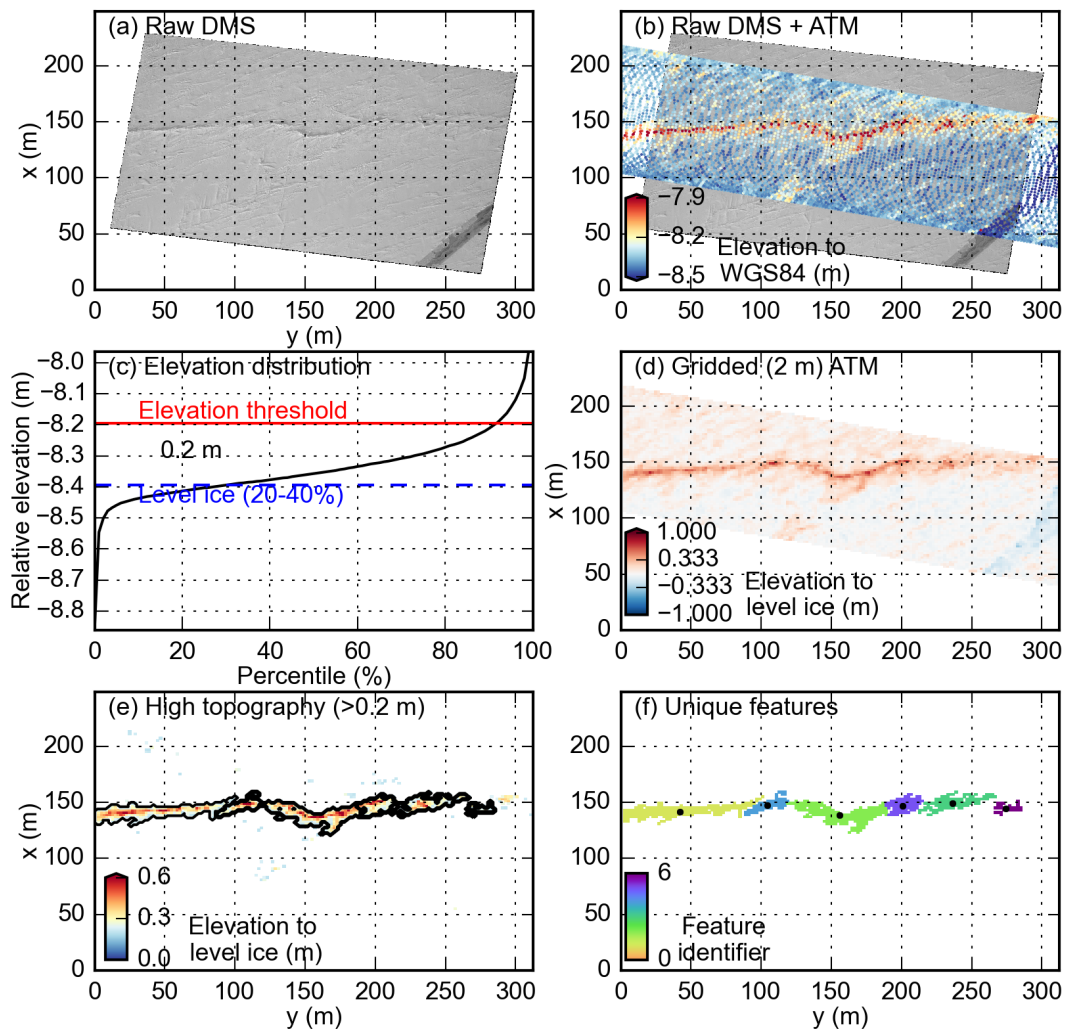

Figure S2. As in Figure S1 but for a DMS image taken on the 23rd March 2011 (in a similar location, as shown by the yellow star in Figure 2) 
DMS Date: 20120322 DMS Time: $14280792 \quad 75.64 \mathrm{~N},-132.68 \mathrm{E}$
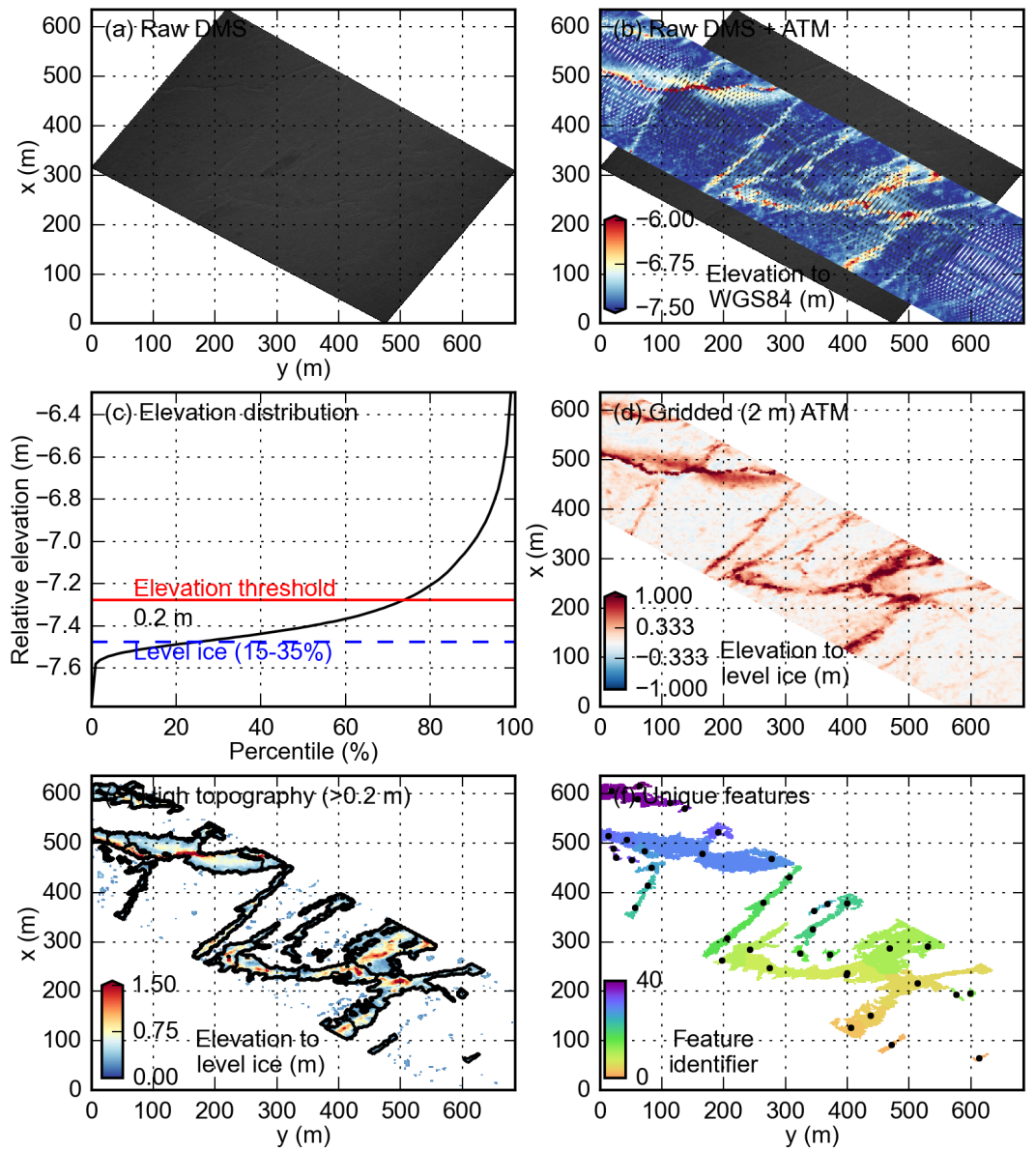

Figure S3. As in Figure S1 but for a DMS image taken on the 22nd March 2012 (blue star in Figure 2) 

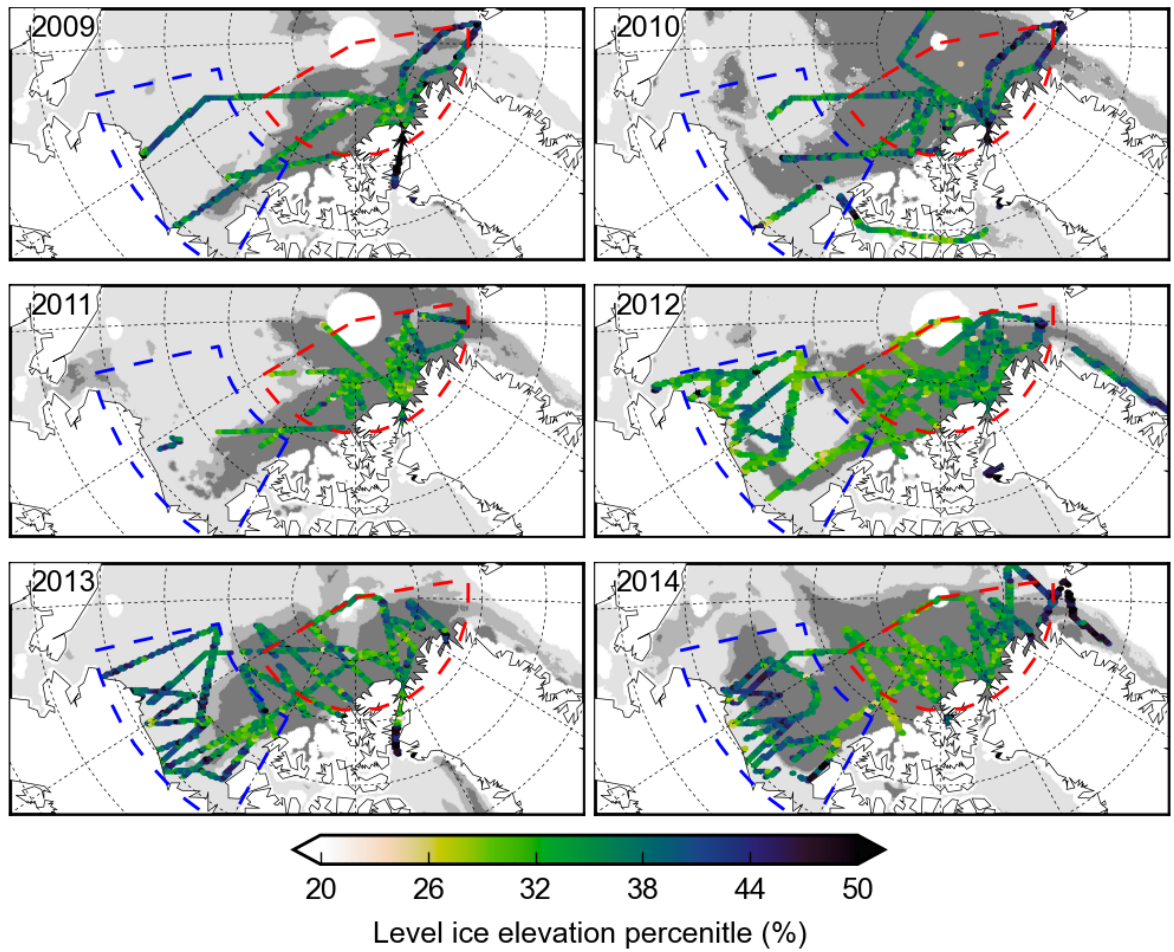

Figure S4. Level ice elevation percentile, from 2009-2014, detected using a $20 \mathrm{~cm}$ elevation threshold. The dark grey (light grey) background indicates regions where more than $80 \%$ of the daily data within each year's IceBridge sea ice campaign dates are estimated as MYI (FYI), while the medium grey indicates a mix of FYI and MYI. The red (blue) dashed lines represent the Central Arctic (Beaufort/Chukchi) regions used in this study. The data are plotted using hexagonal bins. 


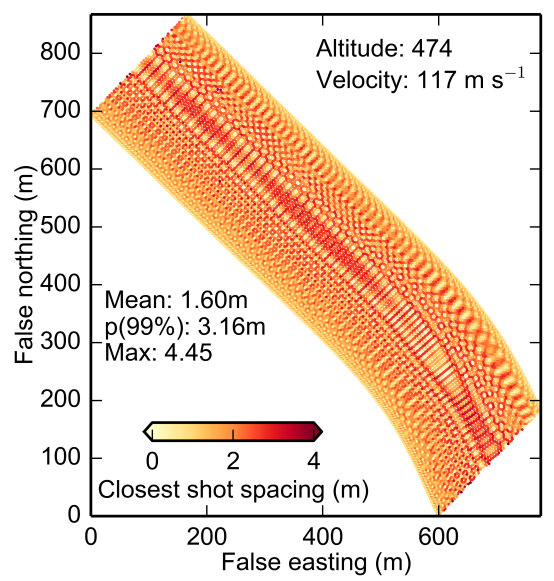

Figure S5. The nearest shot spacing of all ATM points within a $1 \mathrm{~km}$ section, taken from the $1 \mathrm{st}$ April 2011. The mean, 99th percentile and maximum shot spacing are shown, along with the plane altitude and velocity. 


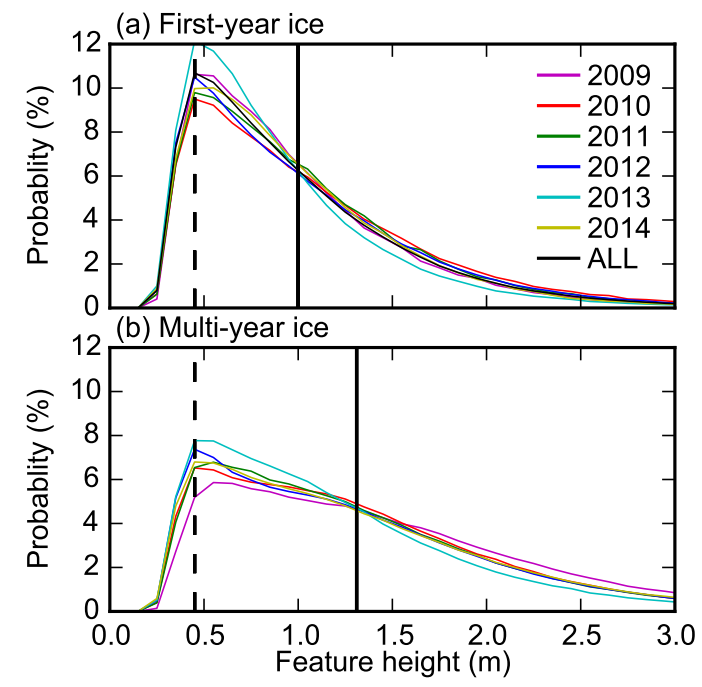

Figure S6. Probability distributions of the surface feature height, $h_{f}$ (using a $20 \mathrm{~cm}$ elevation threshold) detected within the (a) Central Arctic and (b) Beaufort/Chukchi regions (shown in Figure 5). The bin width is $10 \mathrm{~cm}$ and the bin values are plotted as lines (joining each value) instead of steps for clarity. The solid (dashed) vertical lines show the mean (mode) of the distributions across each year. 


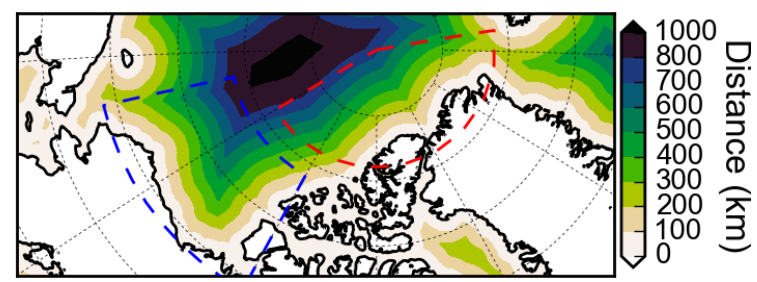

Figure S7. Distance to the nearest coastline (data taken from http://oceancolor.gsfc.nasa.gov/ DOCS/DistFromCoast/.) 